\title{
Study on Clearance Optimization of All-metal Screw Pumps: Experiment and Simulation
}

\author{
Mingzhong LI*, Dong JIANG**, Haibin GUO***, Fanlin MENG****, Meng YU*****, \\ Weixuan DU******,Guodong ZHANG******* \\ *College of Petroleum Engineering, China University of Petroleum, Qingdao 266580, China, E-mail: limzh@upc.edu.cn \\ **College of Petroleum Engineering, China University of Petroleum, Qingdao 266580, China, and Research Institute of \\ Petroleum Engineering, Shengli Oil Field, SINOPEC, Dongying25700, China, E-mail: jiangdong206.slyt@sinopec.com \\ ***Research Institute of Petroleum Engineering, Shengli Oil Field, SINOPEC, Dongying25700, China, \\ E-mail: guohaibin918.slyt@sinopec.com \\ ****College of Mechanical and Electronic Engineering, China University of Petroleum, Qingdao 266580, China, \\ E-mail: mengfanlinlll@foxmail.com \\ *****Oilfield Production $R$ \& D Institute, China Oilfield Services Limited, Tianjin 300450, China, \\ E-mail: ymshida@126.com \\ ******Research Institute of Petroleum Engineering, Shengli Oil Field, SINOPEC, Dongying25700, China, \\ E-mail: duweixuan.slyt@sinopec.com \\ *******College of Petroleum Engineering, China University of Petroleum, Qingdao 266580, China, \\ E-mail: zhang_gd1987@hotmail.com
}

cross $^{\text {ref }}$ http://dx.doi.org/10.5755/j01.mech.23.5.15387

\section{Nomenclature}

$\eta$ - volume efficiency, $Q$ - actual flow rate, $\mathrm{m}^{3} / \mathrm{s}$, $Q t$ - theoretical flow rate, $\mathrm{m}^{3} / \mathrm{s}, Q_{i-j}$ - leakage from high pressure chamber $i(2,3,4,5,6)$ to low pressure chamber $j$ $(1,2,3), \mathrm{m}^{3} / \mathrm{s}, Q l$ - the amount of leakage, $\mathrm{m}^{3} / \mathrm{s}, b$ - clearance seal width, $\mathrm{m}, h$ - clearance height, $\mathrm{m}, \Delta P$ - pressure difference between two ends of clearance, $\mathrm{Pa}, \mu$ - fluid dynamic viscosity, $\mathrm{Pa} \cdot \mathrm{s}, l$ - clearance seal length, $\mathrm{m}$, $D$ - equidistant circular arc diameter of stator line, $\mathrm{m}$.

\section{Introduction}

Since Moineau developed the single-screw hydraulic machinery theory in the $1930 \mathrm{~s}$, single-screw hydraulic machinery pumps and screw motors have been widely applied in the petroleum and chemical areas [1-2]. Ranked as the third artificial lift method, the screw pump has its unique advantages used for lifting heavy oil reservoirs. Conventional screw pumps consist of rubber bushings and metal rotors, which are mainly applied in cold production and lifting of heavy oils or other viscous liquids [3]. In recent years, with the deepening development of ultra-heavy and super-heavy oils, thermal recovery methods of SAGD and CSS steam injections are widely used. Since conventional rubber screw pumps cannot tolerate more than $160^{\circ} \mathrm{C}$, they are not suitable for thermal recovery wells. For these kinds of oil wells, TOTAL and PCM companies used the all-metal screw pumps lift method in two wells in the Joslyn oilfield in Alberta for field tests. The API gravity of the crude oil in the oil field is $8^{\circ}$ and the oil viscosity is $1.7 \times 10^{6} \mathrm{MPa} \cdot \mathrm{s}$ at reservoir conditions [4]. Until the year 2011, a total of 100 all-metal screw pumps have been deployed worldwide in various applications with average run time over two years [5-7].

Z. Chen, et al. in China conducted experimental research on characteristics of all-metal screw pumps, verifying that all-metal screw pumps have a good adaptability in artificial lift of heavy oils [8]. Recent research studies on the optimization on geometry parameters of the screw pumps are mainly focused on conventional single screw pumps, and taking the actual working conditions into considerations, parameters are modified based on the empirical formula and field trials. For example, in order to obtain better output results, the simple pursuit of a larger flow area has been used as the main design purpose [9]. SAVETH [10] set the incompressible fluid flow as the standard to optimize the rotor helix angle, while GAYMARD et al. optimized the structure sizes according to the target flow rate [11].

Optimizations on structure parameters of the allmetal screw pump are a prerequisite for ensuring its performance characteristics. However, based on recent research status at home and abroad, optimization theories and methods on the clearance between the rotor and the stator of all-metal screw pumps are few. This paper presents the clearance optimization of all-metal screw pump with considerations of high temperature and fluid viscosity. The standard testing method is used to analyze the operating characteristics of all-metal screw pumps, and the testing results can provide guidance for the applications of all-metal screw pumps in the field sites.

\section{Clearance optimizations for rotors in all-metal screw pumps}

When the all-metal screw pump works, the metal rotor carries out the eccentric planetary motion in the stator cavity, Thus, compared with the rubber screw pumps, in order to meet the requirements of rotational movement of the rotors in the stator cavity, clearance fit must be used for stators and rotors of all-metal screw pumps. Two main problems will occur during the whole process of using metal screw pumps. First, the planetary motion between the stator and rotor causes wear interference, which affects their life. Second, the work temperature for metal screw pumps is generally between $150-400^{\circ} \mathrm{C}$, but for thermal recovery wells, the work temperature could be up to $400^{\circ} \mathrm{C}$. 
Due to the expansion deformation of metals caused by high temperatures, the matching status between the stators and rotors can be changed, thus influencing its work. Therefore, optimizing the clearance between the stator and rotor is a key point of structure designs for all-metal screw pumps. The static analysis module in ANSYS Workbench software was used to impose heat loads in the stator and rotors. Deformations of the stator and rotors produced by temperature changes are analyzed, and the optimized clearance between the stators and rotors of all-metal screw pumps are finally determined, and results can be used to guide the design and processing process of all-metal screw pumps.

\subsection{Effects of temperatures on clearances}

The ANSYS finite element analysis software was used to simulate temperature effect on the clearance between the stator and rotor. Parameters for this simulation are: ratio of the head numbers is $2: 3$, the stator lead is $420 \mathrm{~mm}$, rotor lead is $280 \mathrm{~mm}$, eccentricity is $4.9 \mathrm{~mm}$, and outer diameter of the stator is $90 \mathrm{~mm}$.

At $400^{\circ} \mathrm{C}$, with initial clearance of $0.1 \mathrm{~mm}$, the thermal expansion deformation of the stator and rotor is shown in Figs. 1 and 2. As can be seen from the figures, deformations occur in the stator and rotor, which are produced by thermal expansions due to the high temperatures. The stator expands when heated, and the cavity volume increases, thus increasing the clearance between the stator and rotor. The minimum deformation is expected to occur at the direction of the minimum diameter.

The rotor also expands when heated, and thus decreases the clearance between the stator and rotor. The maximum deformation occurs at the direction of the maximum diameter.
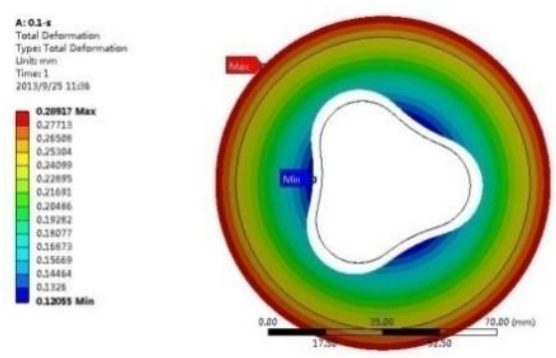

Fig. 1 The deformation of stators

Accordingly, different clearances were analyzed at the condition of $400^{\circ} \mathrm{C}$, the clearances used in this section are $0.02 \mathrm{~mm}, 0.04 \mathrm{~mm}, 0.06 \mathrm{~mm}, 0.08 \mathrm{~mm}, 0.2 \mathrm{~mm}$, and $0.3 \mathrm{~mm}$, and the simulation results are shown in Table 1 .

It is clear from Table 1 that at the temperature of $400^{\circ} \mathrm{C}$, both the inner and outer surfaces of the rotor expand outward due to the effect of heat loads, and when the clearance gap is $0.02 \mathrm{~mm}$, interference fit occurs. The clearance changes greatly for the original clearances of $0.04 \mathrm{~mm}$, and gradually decreases up to $0.3 \mathrm{~mm}$. When the original clearance increases up to $0.3 \mathrm{~mm}$, the relative changing rate of the clearance becomes $7.54 \%$, which is almost unchanged.

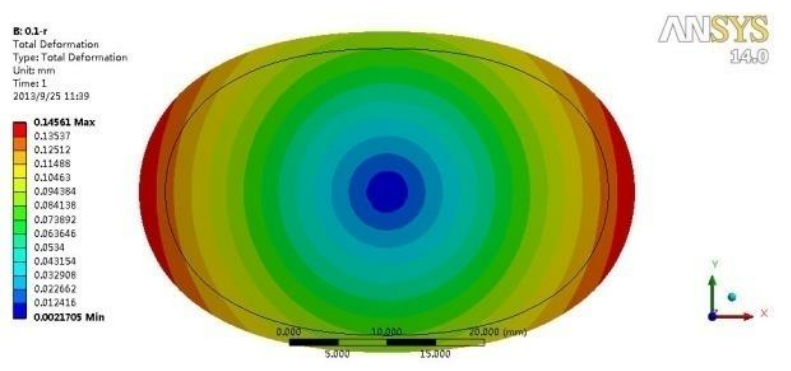

Fig. 2 The deformation of rotors

Table 1

Simulation results of rotor deformation under $400^{\circ} \mathrm{C}$

\begin{tabular}{|c|c|c|c|c|}
\hline $\begin{array}{c}\text { Gap value, } \\
\mathrm{mm}\end{array}$ & $\begin{array}{c}\text { Minimum amount of } \\
\text { deformation of inner- } \\
\text { surface of stators, mm }\end{array}$ & $\begin{array}{c}\text { Maximum defor- } \\
\text { mation of rotor sur- } \\
\text { face, } \mathrm{mm}\end{array}$ & $\begin{array}{c}\text { Clearances after } \\
\text { heating, mm }\end{array}$ & $\begin{array}{c}\text { Relative change } \\
\text { rates of clearanc- } \\
\text { es, } \%\end{array}$ \\
\hline 0.02 & 0.12005 & 0.14561 & -0.00556 & 127.8 \\
0.04 & 0.11923 & 0.14561 & 0.01362 & 65.95 \\
0.06 & 0.12027 & 0.14561 & 0.03466 & 42.23 \\
0.08 & 0.1204 & 0.14561 & 0.05479 & 31.51 \\
0.1 & 0.12179 & 0.14561 & 0.07618 & 23.82 \\
0.2 & 0.12234 & 0.14561 & 0.17673 & 11.63 \\
0.3 & 0.123 & 0.14561 & 0.27739 & 7.54 \\
\hline
\end{tabular}

\subsection{Effects of clearances on pump efficiency}

Fluid flow in stator cavity and the gap between the stator and rotor were simulated using the commercial CFD software Fluent, the variation law of the clearance against pump efficiency was studied, and the influence of medium viscosity and rotational speed were investigated. Finally, the fit clearance between the stator and rotor was optimized.

1. Model establishing and definition of the boundary conditions.

Unsteady model was selected in the calculation.
Realizable $\kappa-\varepsilon$ turbulence model and dynamic mesh model were used to simulate the relative motion of the stator and rotor. Fluid medium was selected as a custom fluid. Equivalent viscosity of a custom fluid was calculated based on the function rate of the crude oil. The well-written CG macro function was loaded to define the border motion.

The geometry model of the fluid computational domain of the stator cavity and the gap are shown in Fig. 3. The outer surface is the 3-spiral-surface with a $420 \mathrm{~mm}$ lead, and the inner surface is 2-spiral-surface with a 280 $\mathrm{mm}$ lead; the minimum clearance between the inner and outer helical surface is $0.9 \mathrm{~mm}$. The eccentric distance of 
the rotor mass is $4.9 \mathrm{~mm}$; the geometry model of the fluid computational domain is meshed. Partially due to the small gap geometry dimension compared to the stator cavity, in order to properly simulate fluid flow characteristics in the gap and take the effect of the grid number on computation into account, local grid refinement is implemented for grids in the gap. While the stator cavity uses grids with larger sizes. The fluid computational domain model is shown in Fig. 4. Due to the large variations of grid sizes for the established three-dimensional mesh model of the fluid computational domain, the tetrahedral grid is used for its better adaptation.

For the established single-stage all-metal screw pump model, according to pressure increase of the allmetal screw pump in the single-stage, the pressure increase of the all-metal screw pump in the single-stage is simulated as $0.5 \mathrm{MPa}$, based on which the inlet and outlet pressures in the model are set as $0.1 \mathrm{MPa}$ and $0.6 \mathrm{MPa}$ respectively.

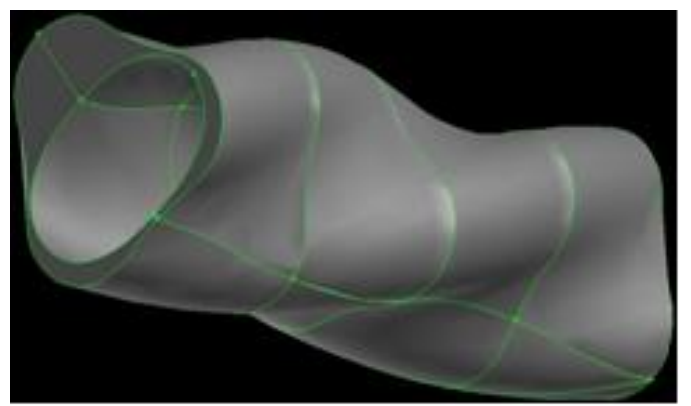

Fig. 3 Geometry model of fluid computational domain

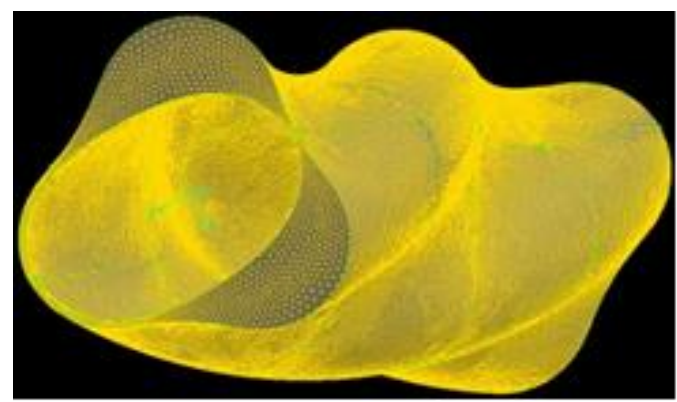

Fig. 4 Grid of fluid computational domain

After all settings, the flow field is initialized, and iteration is conducted until convergence. The calculated velocity field of the clearance and the rotor surface are shown in Figs. 5 and 6, respectively; the calculated velocity vector in the gap and the rotor surface is shown in Figs. 7 and 8 , respectively.

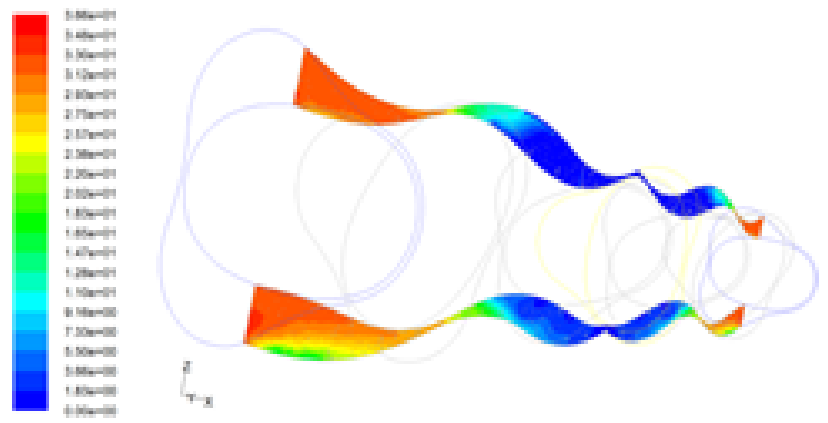

Fig. 5 Velocity profile in the clearance

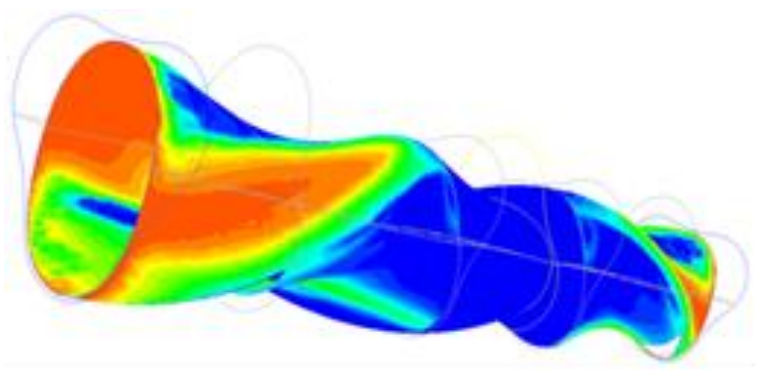

Fig. 6 Velocity profile in the rod surface

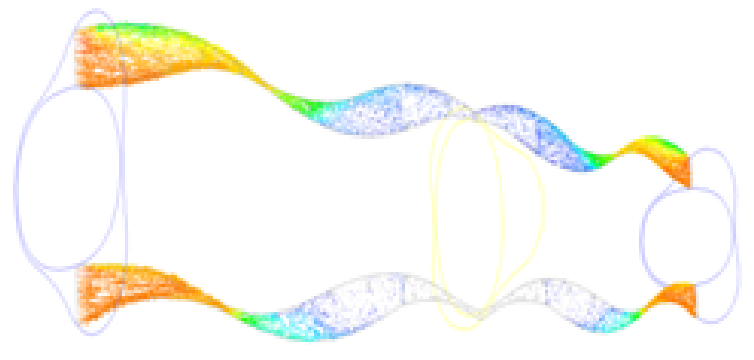

Fig. 7 Velocity vector in the clearance

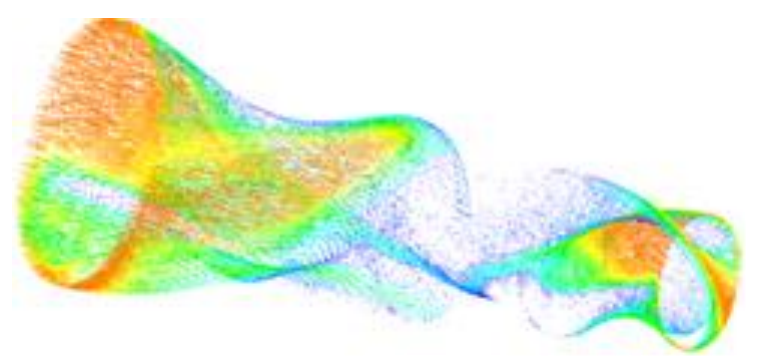

Fig. 8 Velocity vector in the rod surface

2. Sensitivity analyses on the pump efficiency.

The volumetric efficiency of the all-metal screw pump can be calculated using Eq. (1), and it is clear that the volumetric efficiency of the all-metal screw pump depends on flow leakage. Changes in the single-stage pump efficiency of the all metal screw pump are used to analyze the effect of the clearance on the pump efficiency. By using the newly-established model, single-stage leakage flow of the full metal screw pump can be calculated, and then volumetric efficiency of the pump can be calculated by Eq. (1). The volumetric efficiency of this article is represented by the pump efficiency. By changing the value of the clearance and fluid viscosity, changes in the pump efficiency under conditions of different fluid viscosities and variable gaps can be analyzed, and the impact of the clearance on pump efficiency can be further analyzed.

$$
\eta=\frac{Q}{Q_{t}} \times 100 \%=\left(1-\frac{Q_{l}}{Q_{t}}\right) \times 100 \%,
$$

where $\eta$ is volume efficiency; $Q$ is actual flow rate; $Q_{t}$ is theoretical flow rate; $Q_{l}$ is the amount of leakage.

3. Clearance leakage analysis of all-mental screw pump.

Analyzed clearance leakage between stator and rotor whose head number is 2:3. All-mental screw pump drive liquid by engagement of stator and rotor line, pressure increase gradually from inlet to outlet, leaky liquid flow continuously from high-pressure chamber to low- 
pressure chamber. There are two kinds of leakage:

One is caused by linear sealing between chambers, three chambers status are shown in Fig. 9, rotor rotates in stator, engagement forms independent chambers $1,2,3$, corresponding pressure is $P_{1}, P_{2}, P_{3}$. Suppose rotor made a clockwise movement, then $P_{1}>P_{2}>P_{3}$, there exists radial leakage along the axial sealing line from chamber 3 to 2 , chamber 3 to 1 , chamber 2 to 1 , this is pressure differential leakage between chambers and can be calculated by Eq. (2):

$$
Q_{3-2}=Q_{3-1}=Q_{2-1}=\frac{b h^{3} \Delta p}{12 \mu l},
$$

where $b$ is clearance seal width; $h$ is clearance height; $\Delta p$ is pressure difference between two ends of clearance; $\mu$ is fluid dynamic viscosity; $l$ is clearance seal length and is relevant to geometrical dimensions, motion parameters and viscosity of media.

The other is caused by lift seal pressure difference, two chambers status of all-mental screw pump is shown in Fig 10, when rotor reaches $1 / 3$ of stator lead and engages at maximum stator diameter, chamber 1 has finished driving liquid, leave only isolated chamber 2 and 3 driving liquid, circular arc formed by engagement of stator and rotor isolates chamber 1 from next chamber. As rotor continues rotating, chamber 2 and 3 are isolated by circular arc of stator and rotor as well. After completing a lead cycle of motion, liquid in chamber $1,2,3$ is lifted to next chamber $4,5,6$, by repeating this procedure, liquid is lifted from inlet of all-mental screw pump to outlet. Axial pressure difference leakage along equidistant circular arc of stator line from high pressure chamber $4,5,6$ to low pressure chamber $1,2,3$ exists at $1 / 3,2 / 3,3 / 3$ of stator lead. This kind of leakage can be calculated by Eq. (3):

$$
Q_{4-1}=Q_{5-2}=Q_{6-3}=\frac{\pi D h^{3} \Delta p}{12 \mu l} \text {, }
$$

where $D$ is equidistant circular arc diameter of stator line.

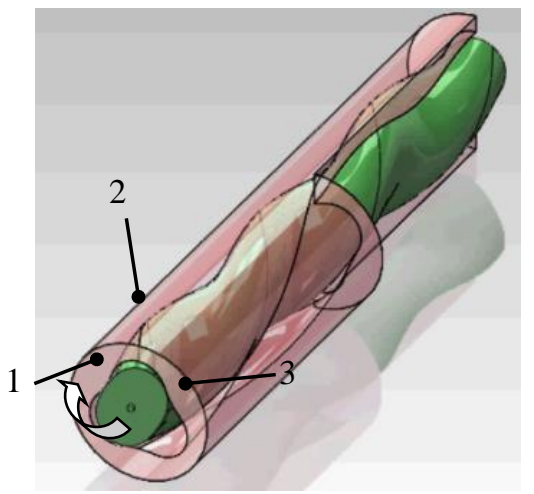

Fig. 9 Three chambers status of all-mental screw pump

When calculating leakage of all-mental screw pump whose head number is $2: 3$, the arc size equals $1 / 3$ circumference of equidistant circular arc, substitute Eq. (3) to get Eq. (4):

$$
Q_{4-1}=Q_{5-2}=Q_{6-3}=\left(\frac{\pi D h^{3} \Delta p}{12 \mu l}\right) / 3=\frac{\pi D h^{3} \Delta p}{36 \mu l}
$$

Take two kinds of leakage into consideration, whole leakage can be calculated by Eq. (5):

$$
\begin{aligned}
& Q_{1}=Q_{3-2}+Q_{3-1}+Q_{2-1}+Q_{4-1}+Q_{5-2}+Q_{6-3}= \\
& =(3 b+\pi D) \frac{h^{3} \Delta p}{12 \mu l} .
\end{aligned}
$$

It is known from Eq. (5) that clearance leakage of all-mental screw pump is proportionate to cube of stator and rotor clearance $h$, also proportionate to pressure difference $\Delta p$ between chambers, is inversely proportional to viscosity of lifting media.

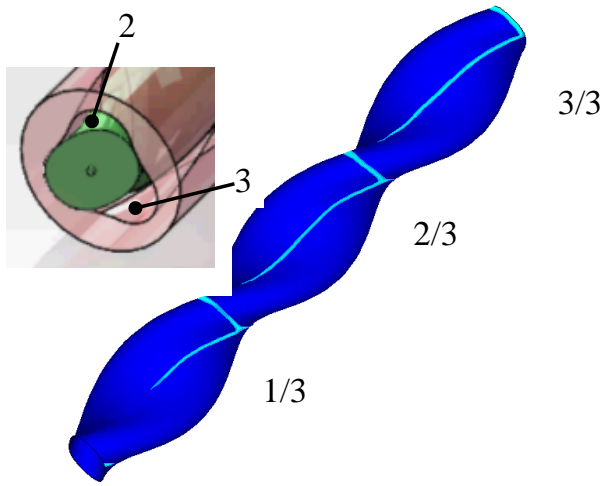

Fig. 10 Two chambers status of all-mental screw pump

The screw pump efficiency is simulated at the rotational speed of $200 \mathrm{rpm} \cdot \mathrm{min}^{-1}$, and the effects of the fluid viscosities and the clearances are analyzed. Simulation results are shown in Fig. 11. It is clear that, when the speed is $200 \mathrm{rpm} \mathrm{min}{ }^{-1}$, the pump efficiency of different fluid viscosities shows decreased inclination with the increase in clearances. For low viscosity fluids $(0.001 \mathrm{~Pa} \mathrm{~s}, 0.005 \mathrm{~Pa} \cdot \mathrm{s}$ and $0.01 \mathrm{~Pa} \cdot \mathrm{s}$ ), a large amount of leakage and low pump efficiency are observed with the increase in clearances. When the clearances are $0.1 \mathrm{~mm}$ and $0.2 \mathrm{~mm}$, the pump efficiency is $60 \%$, while it decreases dramatically when clearances are larger than $0.3 \mathrm{~mm}$. For the fluid viscosity of $0.05 \mathrm{~Pa} \cdot \mathrm{s}, 0.1 \mathrm{~Pa} \cdot \mathrm{s}, 0.2 \mathrm{~Pa} \cdot \mathrm{s}$, the increase in fluid viscosities reduces the fluid flow behavior and increase the leakage friction, and thus pump efficiency decreases slightly with the increase in the clearances, which maintains above $60 \%$.

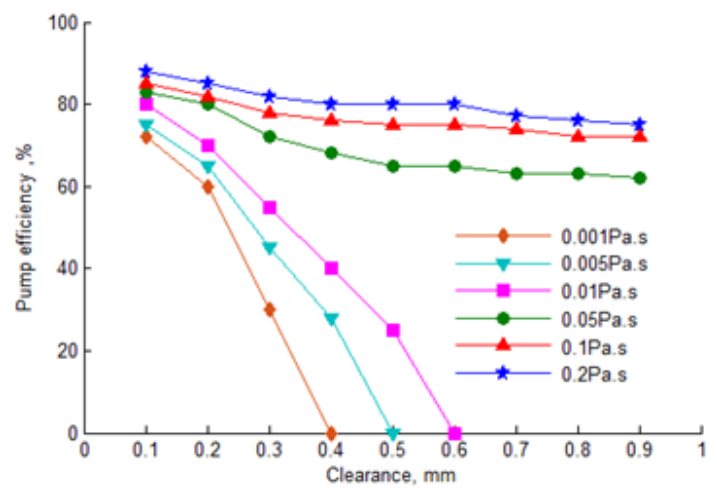

Fig. 11 Effects of clearances and viscosity on the pump efficiency

Simulation results show that the smaller the clearances, the higher the pump efficiency. Since the work con- 
ditions of the all-metal screw pump are high temperature and low viscous fluids, in order to keep the all-metal screw pump work well, the ultimately optimized clearance for the all-metal screw pump is $0.1-0.2 \mathrm{~mm}$.

\subsection{Numerical simulation of external characteristics of the} all-metal screw pump

Based on computational fluid dynamic model in Section 2.2, the effects of different working conditions on the external characteristics of the all-metal screw pumps were studied. For oil and water with viscosity of $0.5 \mathrm{~Pa} \cdot \mathrm{s}$, simulation analysis on the pump efficiency with rotational speed of $200 \mathrm{rpm} \cdot \mathrm{min}^{-1}$ was conducted. The pressure field inner the pump is shown in Fig. 12.

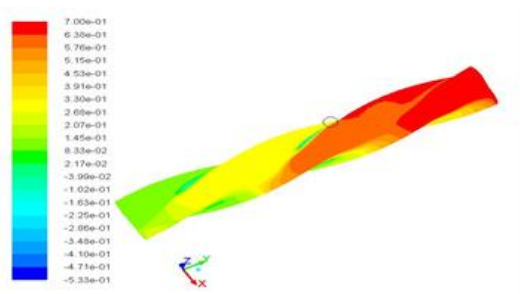

Fig. 12 Pressure field inner the pump

Actual flow simulation of all metal screw pumps under different pressure conditions was studied, and the pump efficiency can be obtained by using Eq. (1). Using the same method and medium, further simulation studies on the pump efficiency for rotational speed of $100 \mathrm{rpm} \cdot \mathrm{min}^{-1}$ and $150 \mathrm{rpm} \cdot \mathrm{min}^{-1}$ were calculated. Varia- tion in the pump efficiency of the all-metal screw pump with pressures and rotational speed are shown in Fig. 13.

Fig. 13 shows that the laws of the pump efficiency differ when transporting oil and water. When oil is chosen as a transport medium, the pump efficiency remains constant with the increase in pressures. When water is used as a transport medium, a substantial decline occurs when increasing pressures. For both of the oil and water transport media, the pump efficiency can be improved by increasing the rotational speed.

The simulation results show that the viscosity and rotational speed are the main factors influencing the pump efficiency of the all-metal screw pump. Since the impact of viscosity on the pump efficiency is greater, the full metal screw pump is more suitable for transport of high viscosity fluids.

\section{Test and analysis of the All-metal screw pumps}

\subsection{Test procedures}

Based on the optimized clearance, a model machine of the all-metal screw pump of GLB160-30-JS was manufactured, and indoor experimental platform was established. The device equipment consists of data collecting and controlling system, electric motor, torque/rotational speed meter, all-metal screw pump, electrical pressure gauge, valves and media container. The testing accurate of the data collecting and controlling system is class B. Pressure precision is $\pm 1.0 \%$. The precision of the flow volume is $\pm 1.5 \%$, and the precision of the rotational speed is \pm 0.2 .

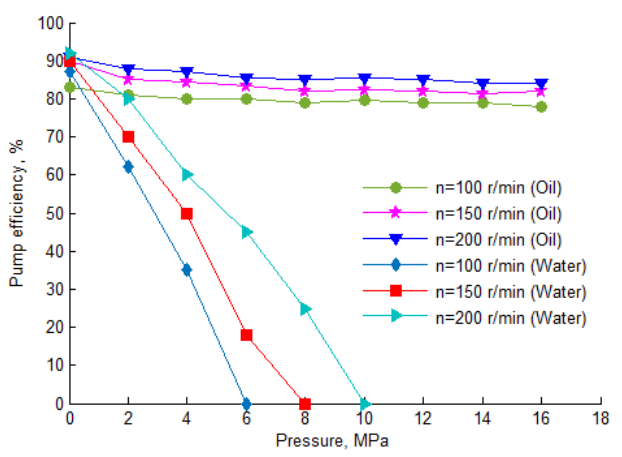

Fig. 13 Variation in the pump efficiency of the all-metal screw pump with pressures and rotational speed

Experimental study was carried on pump efficiency and lift of the all-metal screw pump. The tests were conducted under the follow work conditions: water contents of $0 \%, 10 \%, 20 \%, 30 \%, 40 \%, 50 \%, 60 \%$, and $70 \%$, and rotational speeds of 100,150 , and $200 \mathrm{rpm} \cdot \mathrm{min}^{-1}$.

Tests procedures are as follow.

1. According to Fig. 14, the all-metal screw pump was connected to the experimental platform.

2. The system of data collecting and controlling was used to control the work of the all-metal screw pump. The flow rate was adjusted by the pressure controlling valves, which will change the outlet pressure and flow volume.

3. The system of data collecting and controlling can collect the data automatically, and the data of rotation- al speed, outlet pressure and flow volume were collected continuously.

4. Water and oil were chosen as the test fluids, and the pump efficiency tests were carried out. The water contents used in this test are 10\%, 20\%, 30\%, 40\%, 50\%, $60 \%, 70 \%$, and $100 \%$, respectively.

\subsection{Experimental results}

1. Data processing

The volumetric efficiency of the all-metal screw pump is calculated by Eq. (1), and the relationships between the pump efficiency and head under different media and rotational speeds were drawn.

2. Effects of the rotational speeds on the pump efficiency. 
Pure water and oil were used as test media, under the rotational speeds of 100,150 , and $200 \mathrm{rpm} \cdot \mathrm{min}^{-1}$, to test characteristics of the all-metal screw pump. Test results are shown in Fig. 15. As can be seen from Fig. 13, the allmetal screw pump reflects different characteristics by using different media (water and oil). For water, the overall pump efficiency decreases rapidly, and with the increase in rotational speeds, the pump efficiency declines slowly. When the rotational speed reaches $200 \mathrm{rpm} \cdot \mathrm{min}^{-1}$ and the pump discharge pressure reaches $11 \mathrm{MPa}$, pump efficiency becomes to be zero. For oil, the all-metal screw pumps show good features, and the rotational speeds have little effect on the pump efficiency, and pump efficiencies are more than $75 \%$ for all cases. When the speed is $200 \mathrm{rpm} \cdot \mathrm{min}^{-1}$ and the discharge pressure is $16 \mathrm{MPa}$, the pump efficiency reaches $70 \%$.

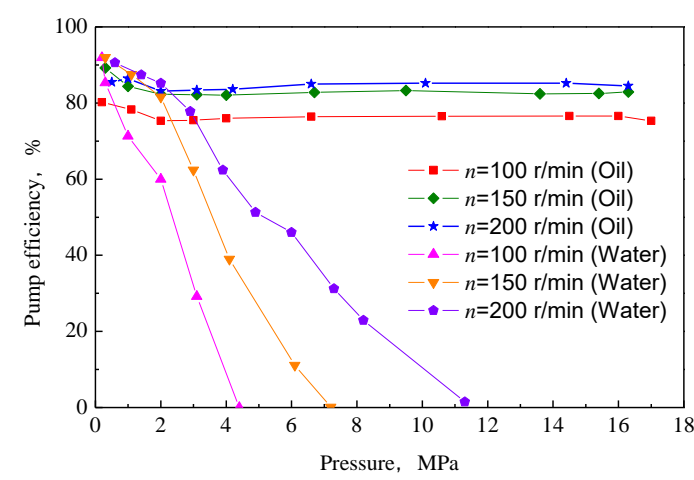

Fig. 15 Characteristic curves of all-metal screw pumps by using pure water/pure oil

cy.

\section{Effects of water contents on the pump efficien-}

Media with different water contents are used to conduct the tests. The viscosity of the testing oil is $53 \mathrm{MPa} \cdot \mathrm{s}$, and the viscosity of the testing water is $1 \mathrm{mPa} \cdot \mathrm{s}$. Tests on fluid viscosity with different water contents are conducted. It is clear from Fig. 16 that when the water content is less than $50 \%$, the testing fluid viscosity shows oil properties, and the viscosity increases with increase in water contents. When the water content is more than $50 \%$, the viscosity of the experimental fluid decreases drastically, indicating water properties. The Richardson correlation [12] of emulsion viscosity was used to fit the experimental results, it is clear from Fig. 16 that the Richardson correlation can fit the experimental data accurately.

For the rotational speed of $150 \mathrm{rpm} \cdot \mathrm{min}^{-1}$, media with different water contents are used to conduct experiments, and results are showed in Fig. 17. It is clear that when the water content is less than $50 \%$, with discharge pressures changing from low to high, the pump efficiency shows a decreasing trend. When the pressure reaches 16.5 $\mathrm{MPa}$, the pump efficiency maintains more than $80 \%$. When the water content is more than $50 \%$, with increase in discharge pressures, the pump efficiency decreases rapidly from its original value (closely to $100 \%$ ), and when the pressure drops to $8 \mathrm{MPa}$, the pump efficiency reduces to zero. Test results are similar with those obtained from pure water and oil.

4. Comparison analyses on characteristics of allmetal screw pumps and rubber stator screw pumps.

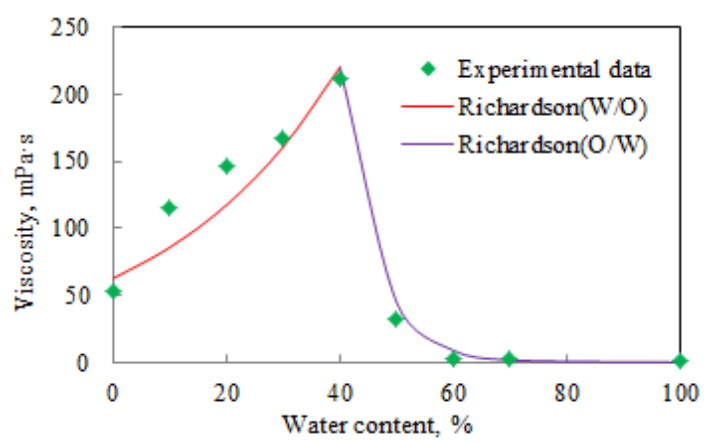

Fig. 16 Viscosity of the fluid with different water contents

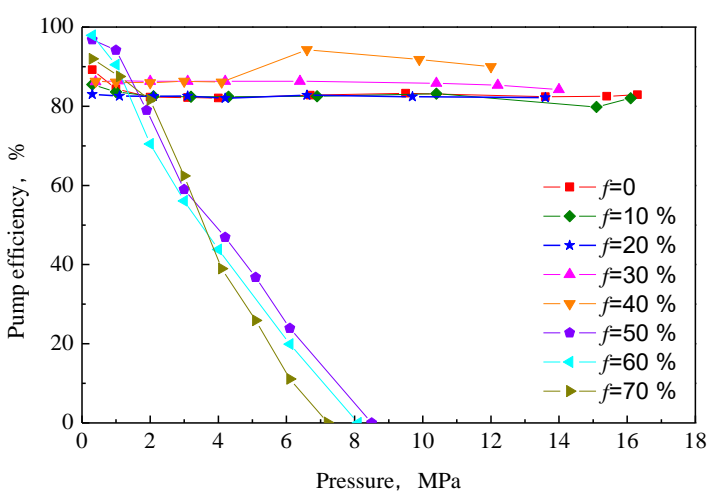

Fig. 17 Relations of the pump efficiency with head at the speed of $150 \mathrm{rpm} \cdot \mathrm{min}^{-1}$ using media of different water contents

To conduct the comparison analysis on the allmetal screw pump and rubber stator screw pump, tests on the outside characteristics are conducted for two rubber stator screw pumps of GLB120-36 and GLB190-33 and one all-metal screw pump of GLB160-30-JS. The testing results are shown in Fig. 18. The rotational speed is $150 \mathrm{rpm} \cdot \mathrm{min}^{-1}$, and water and hydraulic oil are used as medium at the temperature of $30^{\circ} \mathrm{C}$. It is clear from Fig. 18 that the outside characteristics of the all-metal screw pump and rubber stator screw pump are significantly influenced by the media viscosities. For the media of low viscosity water, the metal screw pumps exhibit similar characteristics with the rubber screw pumps. With increase of the discharge pressures, the amount of loss increases. Due to the clearance fit, under the same discharge pressure (head) conditions, the leakage of the metal screw pump is more serious. When the hydraulic oil of $53 \mathrm{MPa} \cdot \mathrm{s}$ is used as a medium, the pump efficiency of the metal screw pumps almost maintains a constant value with the increase in the discharge pressure, indicating an excellent performance.

As analyzed above, the leakage mechanisms of the all-metal screw pump and rubber stator screw pump are different. For rubber stator screw pump, the rubber stator is squeezed to deform, and when the clearance between the stator and rotor exceeds the critical value, the pump is" broken down" and start to leak. While leakage is a natural character of the all-metal screw pump due to the clearance fit. However, the leakage of the all-metal screw pump depends on media viscosities, and the effect of the clearance change induced from pressure on the leakage is neglected. 


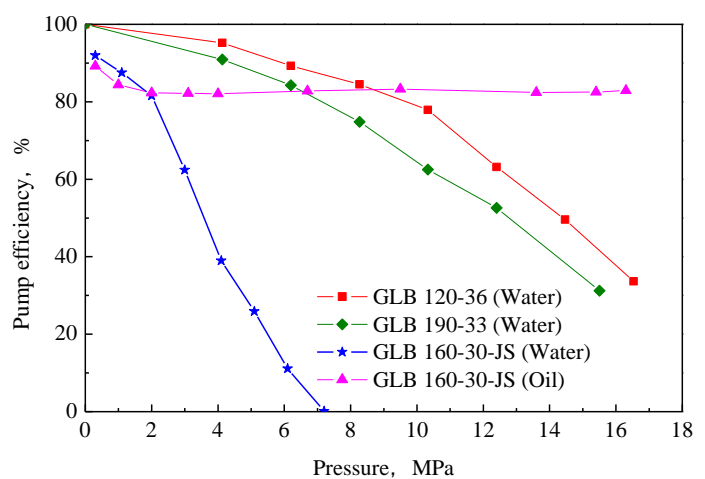

Fig. 18 Variation of pump efficiencies with pressure for metal screw pumps and rubber screw pumps

\subsection{Field test for the all-metal screw pump}

The GLB160-30-JS all-metal screw pump was applied in well 1-15-612 and well 219x1 of Shengli Oilfield in 2015. The application status is shown in Figs. 19 and 20, and its application parameters are shown in Table 2 and Table 3. Field test results show that the all-metal screw pump performance could meet the design requirements with a stable and reliable running condition.

1. Example 1: Well 1-15-612.

The well 1-15-612 is thermal production well of heavy oils with casing of 7 in, reservoir depth of $1315.8 \mathrm{~m}$, reservoir thickness of 7.9 meters. Due to the high water content, the well was shut-in in early production period. Since February 2015, steam stimulation was used for oil recovery, the all-metal screw pump GLB160-30-JS was all equipment has been running smoothly for 14 months and applied for oil production under high temperature conditions. The top drive method and 1 in rod were selected, and the pump efficiency maintains stable conditions with a maximum value of $52 \%$.

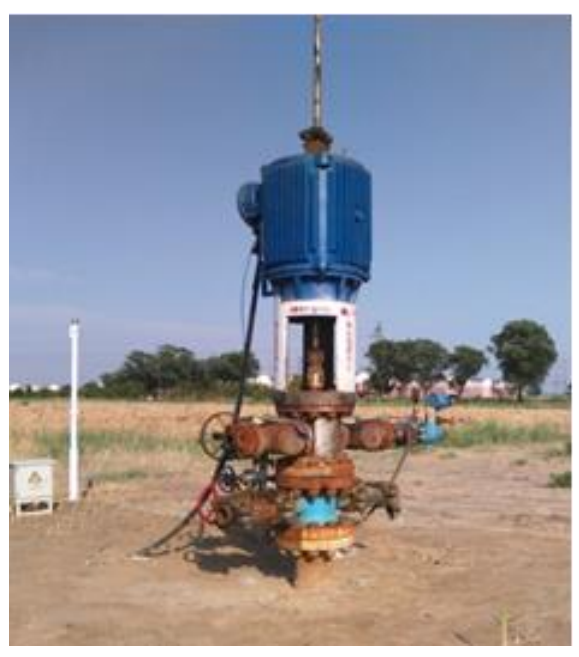

Fig. 19 Field test photo of well 1-15-612

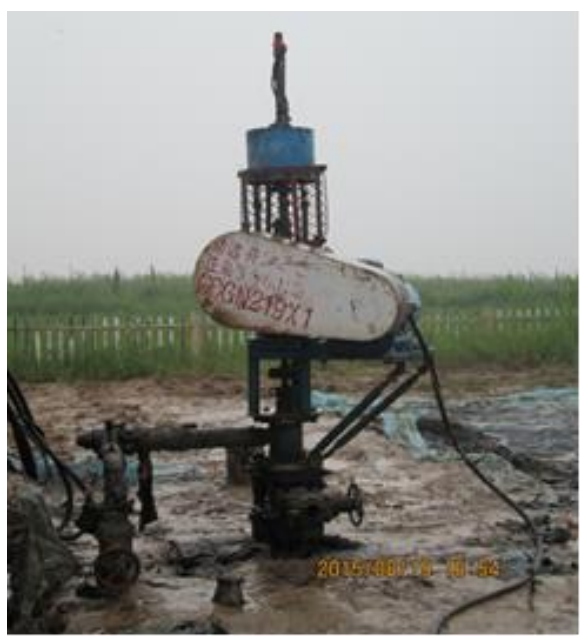

Fig. 20 Field test photo of well 219x1

Parameters of well 1-15-612

Table2

\begin{tabular}{|c|c|c|c|c|}
\hline Pump depth & Rotational speed & Liquid rate & Oil rate & Water content \\
\hline $1100 \mathrm{~m}$ & $140 \mathrm{r} / \mathrm{min}$ & $20 \mathrm{~m}^{3} / \mathrm{d}$ & $1 \mathrm{~m}^{3} / \mathrm{d}$ & $95 \%$ \\
\hline
\end{tabular}

Table3

Parameters of well 219x

\begin{tabular}{|c|c|c|c|c|}
\hline Pump depth & Rotational speed & Liquid rate & Oil rate & Water content \\
\hline $850 \mathrm{~m}$ & $120 \mathrm{r} / \mathrm{min}$ & $17.8 \mathrm{~m}^{3} / \mathrm{d}$ & $8.1 \mathrm{~m}^{3} / \mathrm{d}$ & $54.5 \%$ \\
\hline
\end{tabular}

2. Example 2: Well 219x1.

The casing of the well $219 \mathrm{x}$ is 7 with the reservoir depth of $1502.8 \mathrm{~m}$, reservoir thickness of $6.7 \mathrm{~m}$. Rod pump lift method was selected for the previous production, but the problem of the delayed rod occurs. In June, 2015, steam stimulation was applied, and downhole temperature reaches $140-180^{\circ} \mathrm{C}$. Then the GLB160-30-JS all-metal screw pump was applied. The top drive and a $\Phi 36 \mathrm{~mm}$ hollow rod were chosen. The GLB160-30-JS all-metal screw pump in the well 219x has been smoothly running for 10 months, and a stable pump efficiency remains with a value of $64.5 \%$.

Field applications of the all-metal screw pump in well 1-15-612 and well 219x1 show that, the performance of the designed all-metal screw pump meets the design requirements, and its operating characteristics consistent with the results of indoor tests. The operation process maintains stable and reliable with constant pump efficiency.

\section{Conclusions}

1. Parameters for the all-metal screw pump simulation are: ratio of the head numbers is $2: 3$, the stator lead is $420 \mathrm{~mm}$, rotor lead is $280 \mathrm{~mm}$, eccentricity is $4.9 \mathrm{~mm}$, and outer diameter of the stator is $90 \mathrm{~mm}$. The thermodynamic analyses have been implemented on the stator and rotor of the all-metal screw pumps, and the influence of deformations of the stator and rotor induced from heat on the clearance was studied. Meanwhile, the flow fields in the stator and gap between the stator and rotor were simulated, and the pump efficiency was calculated. According to both of the simulation results, the optimized fit clear- 
ance for all-metal screw pumps used in thermal recovery wells was determined as 0.1-0.2 mm.

2. One model machine of the all-metal screw pump was manufactured, and indoor tests were carried out. Testing results show that, the media viscosity and rotational speed are the main factors that affect the pump efficiency of the all-metal screw pump. With increase in the rotational speed, the pump efficiency increases. Since the effect of the media viscosity on pump efficiency is more obvious, the all-metal screw pump is more suitable to lift high viscos fluids.

3. Compared with rubber stator screw pumps, the performance of the all-metal screw pumps is greater with higher pump efficiency, especially for high viscosity fluids. For low viscosity fluid, due to the clearance fit, leakage of the all-metal screw pumps becomes to be pronounced and the pump efficiency is small.

4. When the all-metal screw pump setting depth was $1100 \mathrm{~m}$, rotating speed was $140 \mathrm{r} / \mathrm{min}$, water content was $94 \%$, field application proved that pumping efficiency was higher than $50 \%$ for 10 months, operation characteristics was essentially in agreement with laboratory experiments, can be apply to high temperature lift of heavy oil thermal recovery wells.

\section{Acknowledgments}

This study was supported by the Program for Changjiang Scholars and Innovative Research Team (IRT1294) in China University of Petroleum (East).

\section{References}

1. Lea, J.F.1993. PCP study focuses on artificial life applications, Petroleum Engineer International 65(9): 1013.

2. Mills, R.A.R. 1994. Progressing cavity oil well PumpsPast, present and future, Journal of Canadian Petroleum Technology 33(4): 5.

3. Wu, B.; Li, X. 2010. The special successful PCP applications in heavy oilfield. In SPE progressing cavity pumps conference. http://dx.doi.org/10.2118/136817-MS.

4. Beauquin, J.L.; Ndinemenu, F.O.; Chalier, G.; Lemay, L.; Seince, L.; Damnjanovic, A. 2007. World's First Metal PCP SAGD Field Test Shows Promising Artificial-Lift Technology for Heavy-Oil Hot Production: Joslyn Field Case. In SPE Annual Technical Conference and Exhibition. http://dx.doi.org/10.2118/110479-MS.

5. Beauquin, J.L.; Boireau, C.; Lemay, L.; Seince, L. 2005. Development status of a metal progressing cavity pump for heavy oil and hot production wells. In SPE International Thermal Operations and Heavy Oil Symposium. http://dx.doi.org/10.2118/97796-MS.

6. Beauquin, J.L.; Chalier G.; Ndinemenu F.; LemayL.; Seince, L.; JahnS. 2008. Metal PCP field trial pushes up pumping window for heavy oil hot production: Joslyn field case. World Heavy Oil Congress, Edmonton, Alberta.

7. Rae, M.; Seince, L.; Mitskopoulos, M. 2011. All metal progressing cavity pumps deployed in SAGD. World Heavy Oil Congress, Edmonton, Alberta.
8. Chen, Z.; Liu, Z.; Yang, W.; Niu, G.; Liu, H.2012. Experimental research on characteristic of full metal screw pump. Oil Drilling \& Production Technology 35(5):65-7. (in Chinese). http://dx.doi.org/10.3969/j.issn.10007393.2012.05.017.

9. Wan, B. 1993.Hydraulic machine with single screw rotor. China University of Petroleum Press. (in Chinese)

10. Saveth, K. J. 1993. Field study of efficiencies between progressing cavity, reciprocating, and electric submersible pumps. In SPE Production Operations Symposium. http://dx.doi.org/10.2118/25448-MS.

11. Gaymard, B.; Puyo, P.1988. The progressive cavity pumps in Europe: Results and new developments. 7th Offshore South East Asia Conference, Singapore. https://doi.org/10.2118/17676-MS.

12. Richardson, E.G. 1950.The formation and flow of emulsion. Journal of colloid science 5(4):404-13. http://dx.doi.org/10.1016/0095-8522(50)90064-X.

Mingzhong Li, Dong Jiang, Haibin Guo, Fanlin Meng, Meng Yu, Weixuan Du, Guodong Zhang

\section{STUDY ON CLEARANCE OPTIMIZATION OF ALL- METAL SCREW PUMPS: EXPERIMENT AND SIMULATION}

S u m m a r y

Due to the advantages of heat and wear resistance, all-metal screw pumps have been commonly applied in field tests in recent years. In this work, the methods of thermodynamic and computational fluid dynamic were applied to analyze the effects of temperature and viscosity on clearance between rotor and stator of the all-metal screw pump. The clearance between stator and rotor of the all-metal screw pump has been optimized. Different model machines of the all-metal screw pump with different clearances have been manufactured, and numerous tests have been carried out in laboratory and fields in order to analyze the effects of rotational speed and viscosity of medium on the characteristics of the all-metal screw pumps. Results show that the performance of all-metal screw pumps is compared with that of rubber screw pumps. Results show that the medium viscosity and rotational speeds have significance effect on pump efficiency. An increase in the pump efficiency could be achieved with an increase in the rotational speed. However, compared with the rotational speeds, the viscosity of medium shows a more severe influence on the pump efficiency. Characteristics of the newly developed all-metal screw pumps could satisfy design requirements. Characteristics of the all-metal screw pumps consistent with the results of laboratory experiments, and operation processes are smooth and reliable. This article provides a theoretical guidance to the promotion and application of this technology.

Keywords: all-metal screw pumps, stator and rotor clearance, pump efficiency, clearance optimization.

Received July 12, 2016

Accepted October 13, 2017 